\title{
Incidence of San Jose Scale, (Quadraspidiotus perniciosus Comstock) in Relation to Abiotic Factors on Apple Tree
}

\author{
Insha Hamid $^{1 *}$, A.R. Wani ${ }^{1}$, M.A. Sofi ${ }^{1}$, S.S. Pathania ${ }^{1}$, Nageena nazir ${ }^{2}$, \\ Mohd. Saleem Mir ${ }^{3}$ and G.I. Hassan ${ }^{4}$
}

\begin{abstract}
${ }^{1}$ Division of Entomology, ${ }^{2}$ Division of Agricultural Statistics, ${ }^{3}$ Directorate Research, ${ }^{4}$ Division of RCRQA, Sher-e-Kashmir University of Agricultural Science \& Technology, Kashmir, (J. \& K.), India

*Corresponding author
\end{abstract}

\section{Keywords}

San Jose scale, Quadraspidiotus perniciosus, Abiotic factors Apple tree

Article Info

Accepted:

12 December 2020

Available Online:

10 January 2021
Studies on the incidence of San Jose scale, Quadraspidiotus perniciosus (Comstock) was undertaken in Baramulla and Budgam districts of Kashmir valley. Highest mean per cent incidence of 32.06 was recorded in Baramulla, while as, lowest 25.65 per cent was recorded in district Budgam. Highest mean per cent incidence of 37.85 was observed in low elevation of district Baramulla followed by 31.33 per cent in low elevation of district Budgam. A positive and significant correlation was found between incidence with maximum temperature, maximum relative humidityand sunshine hours in district Baramulla and Budgam. Multiple regression analysis revealed maximum temperature contribute maximum to incidence followed by maximum relative humidity and sunshine hours in both the districts.

\section{Introduction}

Apple, Malus domestica (Borkh) belonging to family Rosaceae is undoubtedly the most important fruit, distributed worldwide and plays an important role in nation's economy. The area under apple cultivation in India is 311.91 thousand hectares with an annual production of 2286.37 thousand metric tonnes; and the productivity of 7.33 metric tonnes per hectare (Anonymous, 2018). The Union Territory of Jammu and Kashmir leads both in acreage and production of apple in the country; with an area and annual production of 164.14 thousand hectares and 187.90 thousand metric tonnes, respectively, at the productivity rate of 11.44 metric tonnes per hectare. The territory has been declared as 
Agro-export zone for apple production, so the priorities have to be shifted from quantity to quality and value addition aspects (Anonymous, 2019). The yield of apple, its quality as well as plant growth is influenced by many environmental factors. However, the apple quality and quantity is subjected to deterioration, which could be attributed to many factors; which among others also include insect pests and diseases. The most important insect pest infesting the apple crop is San Jose Scale, SJS (Quadras pidiotus perniciosus Comstock), associated with almost every apple orchard in every nook and corner of the territory (Rather and Masoodi, 1994). It is especially serious in temperate and sub-tropical regions and occurs in all the temperate fruit producing countries of the world. SJS female gives birth to living young from the eggs developed within the body of the female called immature forms, "crawlers". Damage is caused by feeding of crawlers, which suck plant sap, weaken the plant, reduce fruit and shoot growth and desiccate foliage.

The area under the feeding site turns to characteristic purplish red color. Under high population, the crawlers may migrate from fruit spurs onto the fruit and at harvest the fruits have distinct "measles" spots on the surface and when the scale is removed from the fruit, a high coloured bull's eye is evident (Balusu, 2017). SJS infestation on twigs and limbs can cause an overall decline in tree vigour, growth, foliage, productivity. In most cases, the scale had spread to a serious degree before it was discovered and a greater number of trees are badly injured and killed by its attack. This pest can produce several scales in one season. If uncontrolled, they can kill the tree as well as make the fruit unmarketable. Despite of, extensive work carried in India and abroad on the SJS, a thorough investigation in Kashmir division is lacking. Keeping in view the economic importance of the pest, it is imperative to study the incidence of the pest.

\section{Materials and Methods}

Extensive field surveys were done to ascertain the incidence of SJS during the year 20182019 in two districts Baramulla and Budgam of Kashmir province. Each selected district was categorized in three different altitudes (high, medium and low) (Table-1); from each altitude two villages and from each village two orchards were selected. Three trees were randomly selected from each selected orchard. A total of 36 apple trees from each district was screened for the incidence of SJS.

\section{Pest incidence assessment}

The pest incidence of SJS was recorded by randomly selecting three apple trees from each orchard as detailed in Table 1. Observations regarding the pest incidence were recorded at fortnight interval. The per cent pest incidence was calculated as the proportion of infested apple trees to the total number of trees assessed by the following formula:

$$
\mathrm{PPI}=\frac{\mathrm{n}}{\mathrm{N}} \times 100
$$

Where,

$$
\begin{array}{lll}
\mathrm{PPI} & = & \text { Per cent Pest Incidence } \\
\mathrm{n} & = & \text { Number of infested trees } \\
\mathrm{N} & = & \text { Total number of assessed trees }
\end{array}
$$

\section{Influence of abiotic factors on SJS}

The simple correlation and multiple regression studies between pest incidence with all abiotic factors (temperature, relative humidity, rainfall and sunshine) were worked out so as to determine their impact on pest incidence on apple crop. 


\section{Results and Discussion}

\section{Location wise pest incidence}

Studies conducted to ascertain the incidence at Baramulla and Budgam during 2018-2019 revealed that in all selected villages of district Baramulla, activity of SJS were observed from last week of February $\left(8^{\text {th }} \mathrm{SW}\right)$. Thereafter, a increase in incidence was recorded and attained its peak in last week of July to first week of August ( $30^{\text {th }}$ to $32^{\text {nd }} \mathrm{SW}$ ) and then the incidence declined until last week of December $\left(52^{\text {nd }} \mathrm{SW}\right)$. The cumulative mean incidence of SJS spanning for all 19 weeks of investigation was recorded highest as 37.85 per cent at low altitude and 27.89 per cent as lowest at high altitude; while as 30.43 per cent at medium altitude was recorded (Table 2). The present findings are in close proximity with the work of Botha (2008).

Perusal of data in Table- 3 depicted that in all selected villages of district Budgam, activity of the pest was observed from last week of February $\left(8^{\text {th }} \mathrm{SW}\right)$, which then increased and attained a peak incidence in second week of July to first week of September ( $28^{\text {th }}$ to $36^{\text {th }}$ SW). Thereafter, the incidence declined until last week of December $\left(52^{\text {nd }} \mathrm{SW}\right)$. The cumulative mean incidence of SJS was recorded highest as 31.33 per cent at low altitude and 22.09 per cent as lowest at high altitude; while as 23.54 per cent at medium altitude was observed. The present findings are more or less supported by the work of Botha (2008).

\section{Elevation and month wise pest incidence}

The data was recorded from different elevations of district Baramulla and Budgam in different months. Perusal of data in Table-4 revealed that in district Baramulla the overall highest mean SJS incidence of 38.60 per cent was recorded at low altitude followed by 30.96 per cent at medium altitude, while as at higher altitude it was 28.39 per cent. However, at all three altitudes, the mean pest incidence differed significantly from each other. For all three altitudes the mean per cent incidence in district Baramulla was observed as highest as 55.19 per cent in the month of August followed by 50.58 per cent in July, whereas, in February, March, April and December, the lowest mean incidence of 16.66 per cent was recorded. However, the mean pest incidence in February, March, April and December was statistically at par with each other and differed significantly from the other months. The present findings are more or less supported by the work of (Bandey et al., 2008).

Perusal of data in Table-5 depicted that the mean pest incidence in district Budgam at low altitude was 31.93 per cent followed by 23.74 per cent at medium altitude, while as 22.07per cent at higher altitude was observed. However, at all three altitudes, the mean pest incidence differed significantly from each other. For all the three altitudes the mean per cent incidence was recorded highest as 38.88 per cent in the month of August followed by 38.42 per cent in July whereas, the lowest mean incidence of 16.66 per cent was recorded in February, March, April and December.

However, the mean pest incidence in July and August was statistically at par with each other which differed significantly from remaining months. While as, in May and November the mean pest incidence was statistically at par with each other and differed significantly from remaining months. Similarly, in February, March, April and December the mean incidence was statistically at par with each other. The present findings are more or less supported by the work of (Bandey et al., 2008). 


\section{Correlation and regression studies}

Perusal of Table-6indicated that in the investigation, the simple correlation between various abiotic factors with incidence of a pest revealed a positive and significant correlation between incidence with maximum temperature, maximum relative humidity and sunshine hours. The present study is in conformity with the work of (Supriadi et al.,
2015).

Perusal of Table- 7 and 8 depicted that the multiple regression analysis between various abiotic factors with incidence of a pest revealed that maximum temperature contribute maximum to incidence followed by maximum relative humidity and sunshine hours. The present study is in conformity with the work of Supriadi et al., (2015).

Table.1 Classification of villages on the basis of elevation in two different districts

\begin{tabular}{|l|l|l|}
\hline District & Elevation & Villages \\
\hline & $\begin{array}{l}\text { High } \\
(1700-1800 \mathrm{~m} \text { asl })\end{array}$ & Fatehgadh \\
\cline { 2 - 3 } & $\begin{array}{l}\text { Medium } \\
(1600-1700 \mathrm{~m} \text { asl })\end{array}$ & Badmul \\
\hline \multirow{2}{*}{ Budgam } & $\begin{array}{l}\text { Low } \\
(1500-1600 \mathrm{~m} \text { asl })\end{array}$ & Yaal \\
\hline & $\begin{array}{l}\text { High } \\
(1800-1900 \mathrm{~m} \text { asl })\end{array}$ & Singpora \\
\hline & $\begin{array}{l}\text { Medium } \\
(1700-1800 \mathrm{~m} \text { asl })\end{array}$ & Kalpora \\
\hline & $\begin{array}{l}\text { Low } \\
(1600-1700 \mathrm{~m} \text { asl })\end{array}$ & Kambalhar \\
\hline & Krimshore \\
\hline
\end{tabular}


Table.2 Per cent incidence of San Jose scale (Quadraspidiotus perniciosus Comstock) on apple at different locations in district Baramulla during 2018-2019

\begin{tabular}{|c|c|c|c|c|c|c|c|c|c|c|c|c|c|c|c|c|c|c|c|c|c|c|c|c|c|c|}
\hline \multirow[t]{2}{*}{ Location } & \multirow{2}{*}{$\begin{array}{l}\text { Altit } \\
\text { ude }\end{array}$} & \multicolumn{23}{|c|}{ Standard weeks } & \multirow{2}{*}{$\begin{array}{c}\text { Mean } \\
(\%) \\
\pm \text { SE }\end{array}$} & \multirow{2}{*}{$\begin{array}{c}\text { Cumalative } \\
\text { pooled mear } \\
(\%) \\
\pm \mathrm{SE}\end{array}$} \\
\hline & & $8^{\text {th }}$ & $\begin{array}{c}10^{\mathrm{t}} \\
\mathrm{h}\end{array}$ & $\begin{array}{c}12^{t} \\
h\end{array}$ & $\begin{array}{c}14^{\mathrm{t}} \\
\mathrm{h}\end{array}$ & $16^{t}$ & $\begin{array}{c}18^{t} \\
h\end{array}$ & $\begin{array}{c}20^{t} \\
h\end{array}$ & $22^{n}$ & $24^{t}$ & $\underset{h}{26^{t}}$ & $\underset{\mathrm{h}}{28^{\mathrm{t}}}$ & $\underset{h}{30^{t}}$ & $\underset{d}{32^{n}}$ & $\begin{array}{c}34^{\mathrm{t}} \\
\mathrm{h}\end{array}$ & $\begin{array}{c}36^{t} \\
h\end{array}$ & $\begin{array}{c}38^{\mathrm{t}} \\
\mathrm{h}\end{array}$ & $\begin{array}{c}40^{t} \\
\mathrm{~h}\end{array}$ & $42^{n}$ & $\begin{array}{c}44^{t} \\
h\end{array}$ & $\begin{array}{c}46^{\mathrm{t}} \\
\mathrm{h}\end{array}$ & $\begin{array}{c}48^{t} \\
h\end{array}$ & $\begin{array}{c}50^{t} \\
h\end{array}$ & $52^{n}$ & & \\
\hline $\begin{array}{c}\text { FATEH } \\
\text { GADH }\end{array}$ & \multirow[t]{2}{*}{$(\mathrm{H})$} & $\begin{array}{l}16 . \\
66^{*}\end{array}$ & $\begin{array}{l}16 . \\
66\end{array}$ & $\begin{array}{l}16 . \\
66\end{array}$ & $\begin{array}{l}16 . \\
66\end{array}$ & $\begin{array}{l}16 . \\
66\end{array}$ & $\begin{array}{l}16 . \\
66\end{array}$ & $\begin{array}{l}24 . \\
99\end{array}$ & $\begin{array}{l}33 . \\
33\end{array}$ & $\begin{array}{l}33 . \\
33\end{array}$ & $\begin{array}{l}33 . \\
33\end{array}$ & $\begin{array}{l}41 . \\
66\end{array}$ & $\begin{array}{l}50 . \\
00\end{array}$ & $\begin{array}{l}50 . \\
00\end{array}$ & $\begin{array}{l}50 . \\
00\end{array}$ & $\begin{array}{l}33 . \\
33\end{array}$ & $\begin{array}{l}33 . \\
33\end{array}$ & $\begin{array}{l}33 . \\
33\end{array}$ & $\begin{array}{l}33 . \\
33\end{array}$ & $\begin{array}{l}33 . \\
33\end{array}$ & $\begin{array}{l}16 . \\
66\end{array}$ & $\begin{array}{l}16 . \\
66\end{array}$ & $\begin{array}{l}16 . \\
66\end{array}$ & $\begin{array}{l}16 . \\
66\end{array}$ & $\begin{array}{l}28.25 \\
\pm 2.49\end{array}$ & \multirow[t]{2}{*}{$27.89 \pm 0.36$} \\
\hline $\begin{array}{c}\text { BADMU } \\
\text { L }\end{array}$ & & $\begin{array}{l}16 . \\
66\end{array}$ & $\begin{array}{l}16 . \\
66\end{array}$ & $\begin{array}{l}16 . \\
66\end{array}$ & $\begin{array}{l}16 . \\
66\end{array}$ & $\begin{array}{l}16 . \\
66\end{array}$ & $\begin{array}{l}16 . \\
66\end{array}$ & $\begin{array}{l}24 . \\
99\end{array}$ & $\begin{array}{l}33 . \\
33\end{array}$ & $\begin{array}{l}33 . \\
33\end{array}$ & $\begin{array}{l}33 . \\
33\end{array}$ & $\begin{array}{l}33 . \\
33\end{array}$ & $\begin{array}{l}50 . \\
00\end{array}$ & $\begin{array}{l}50 . \\
00\end{array}$ & $\begin{array}{l}50 . \\
00\end{array}$ & $\begin{array}{l}33 . \\
33\end{array}$ & $\begin{array}{l}33 . \\
33\end{array}$ & $\begin{array}{l}33 . \\
33\end{array}$ & $\begin{array}{l}33 . \\
33\end{array}$ & $\begin{array}{l}24 . \\
99\end{array}$ & $\begin{array}{l}16 . \\
66\end{array}$ & $\begin{array}{l}16 . \\
66\end{array}$ & $\begin{array}{l}16 . \\
66\end{array}$ & $\begin{array}{l}16 . \\
66\end{array}$ & $\begin{array}{l}27.53 \\
\pm 2.42\end{array}$ & \\
\hline BURAN & \multirow[t]{2}{*}{ (M) } & $\begin{array}{l}16 . \\
66\end{array}$ & $\begin{array}{l}16 . \\
66\end{array}$ & $\begin{array}{l}16 . \\
66\end{array}$ & $\begin{array}{l}16 . \\
66\end{array}$ & $\begin{array}{l}16 . \\
66\end{array}$ & $\begin{array}{l}16 . \\
66\end{array}$ & $\begin{array}{l}33 . \\
33\end{array}$ & $\begin{array}{l}33 . \\
33\end{array}$ & $\begin{array}{l}41 . \\
66\end{array}$ & $\begin{array}{l}41 . \\
66\end{array}$ & $\begin{array}{l}50 . \\
00\end{array}$ & $\begin{array}{l}50 . \\
00\end{array}$ & $\begin{array}{l}50 . \\
00\end{array}$ & $\begin{array}{l}50 . \\
00\end{array}$ & $\begin{array}{l}41 . \\
66\end{array}$ & $\begin{array}{l}33 . \\
33\end{array}$ & $\begin{array}{l}33 . \\
33\end{array}$ & $\begin{array}{l}33 . \\
33\end{array}$ & $\begin{array}{l}33 . \\
33\end{array}$ & $\begin{array}{l}33 . \\
33\end{array}$ & $\begin{array}{l}16 . \\
66\end{array}$ & $\begin{array}{l}16 . \\
66\end{array}$ & $\begin{array}{l}16 . \\
66\end{array}$ & $\begin{array}{l}30.79 \\
\pm 2.69\end{array}$ & \multirow[t]{2}{*}{$30.43 \pm 0.36$} \\
\hline YAAL & & $\begin{array}{l}16 . \\
66\end{array}$ & $\begin{array}{l}16 . \\
66\end{array}$ & $\begin{array}{l}16 . \\
66\end{array}$ & $\begin{array}{l}16 . \\
66\end{array}$ & $\begin{array}{l}16 . \\
66\end{array}$ & $\begin{array}{l}16 . \\
66\end{array}$ & $\begin{array}{l}33 . \\
33\end{array}$ & $\begin{array}{l}33 . \\
33\end{array}$ & $\begin{array}{l}33 . \\
33\end{array}$ & $\begin{array}{l}41 . \\
66\end{array}$ & $\begin{array}{l}50 . \\
00\end{array}$ & $\begin{array}{l}50 . \\
00\end{array}$ & $\begin{array}{l}50 . \\
00\end{array}$ & $\begin{array}{l}50 . \\
00\end{array}$ & $\begin{array}{l}41 . \\
66\end{array}$ & $\begin{array}{l}33 . \\
33\end{array}$ & $\begin{array}{l}33 . \\
33\end{array}$ & $\begin{array}{l}33 . \\
33\end{array}$ & $\begin{array}{l}33 . \\
33\end{array}$ & $\begin{array}{l}24 . \\
99\end{array}$ & $\begin{array}{l}16 . \\
66\end{array}$ & $\begin{array}{l}16 . \\
66\end{array}$ & $\begin{array}{l}16 . \\
66\end{array}$ & $\begin{array}{l}30.06 \\
\pm 2.65\end{array}$ & \\
\hline $\begin{array}{c}\text { SINGPO } \\
\text { RA }\end{array}$ & \multirow[t]{2}{*}{ (L) } & $\begin{array}{l}16 . \\
66\end{array}$ & $\begin{array}{l}16 . \\
66\end{array}$ & $\begin{array}{l}16 . \\
66\end{array}$ & $\begin{array}{l}16 . \\
66\end{array}$ & $\begin{array}{l}16 . \\
66\end{array}$ & $\begin{array}{l}33 . \\
33\end{array}$ & $\begin{array}{l}41 . \\
66\end{array}$ & $\begin{array}{l}50 . \\
00\end{array}$ & $\begin{array}{l}58 . \\
33\end{array}$ & $\begin{array}{l}66 . \\
66\end{array}$ & $\begin{array}{l}66 . \\
66\end{array}$ & $\begin{array}{l}66 . \\
66\end{array}$ & $\begin{array}{l}66 . \\
66\end{array}$ & $\begin{array}{l}66 . \\
66\end{array}$ & $\begin{array}{l}50 . \\
00\end{array}$ & $\begin{array}{l}50 . \\
00\end{array}$ & $\begin{array}{l}33 . \\
33\end{array}$ & $\begin{array}{l}33 . \\
33\end{array}$ & $\begin{array}{l}33 . \\
33\end{array}$ & $\begin{array}{l}33 . \\
33\end{array}$ & $\begin{array}{l}16 . \\
66\end{array}$ & $\begin{array}{l}16 . \\
66\end{array}$ & $\begin{array}{l}16 . \\
66\end{array}$ & $\begin{array}{l}38.40 \\
\pm 4.14\end{array}$ & \multirow[t]{2}{*}{$37.85 \pm 0.54$} \\
\hline $\begin{array}{c}\text { KISANB } \\
\text { AGH }\end{array}$ & & $\begin{array}{l}16 . \\
66\end{array}$ & $\begin{array}{l}16 . \\
66\end{array}$ & $\begin{array}{l}16 . \\
66\end{array}$ & $\begin{array}{l}16 . \\
66\end{array}$ & $\begin{array}{l}16 . \\
66\end{array}$ & $\begin{array}{l}33 . \\
33\end{array}$ & $\begin{array}{l}33 . \\
33\end{array}$ & $\begin{array}{l}50 . \\
00\end{array}$ & $\begin{array}{l}58 . \\
33\end{array}$ & $\begin{array}{l}58 . \\
33\end{array}$ & $\begin{array}{l}66 . \\
66\end{array}$ & $\begin{array}{l}66 . \\
66\end{array}$ & $\begin{array}{l}66 . \\
66\end{array}$ & $\begin{array}{l}58 . \\
33\end{array}$ & $\begin{array}{l}50 . \\
00\end{array}$ & $\begin{array}{l}50 . \\
00\end{array}$ & $\begin{array}{l}33 . \\
33\end{array}$ & $\begin{array}{l}33 . \\
33\end{array}$ & $\begin{array}{l}33 . \\
33\end{array}$ & $\begin{array}{l}33 . \\
33\end{array}$ & $\begin{array}{l}16 . \\
66\end{array}$ & $\begin{array}{l}16 . \\
66\end{array}$ & $\begin{array}{l}16 . \\
66\end{array}$ & $\begin{array}{l}37.31 \\
\pm 3.95\end{array}$ & \\
\hline $\begin{array}{l}\text { MEAN } \\
(\%) \pm \text { SE }\end{array}$ & & $\begin{array}{c}16 . \\
66 \\
\pm 0 . \\
00\end{array}$ & $\begin{array}{r}16 . \\
66 \\
\pm 0 . \\
00\end{array}$ & $\begin{array}{c}16 . \\
66 \\
\pm 0 \\
00\end{array}$ & $\begin{array}{r}16 . \\
66 \\
\pm 0 . \\
00\end{array}$ & $\begin{array}{l}16 . \\
66 \\
\pm 0 . \\
00\end{array}$ & $\begin{array}{r}22 . \\
21 \\
\pm 3 . \\
51\end{array}$ & $\begin{array}{l}31 . \\
94 \\
\pm 2 . \\
56\end{array}$ & $\begin{array}{r}38 . \\
88 \\
\pm 3 . \\
51\end{array}$ & $\begin{array}{r}43 \\
05 \\
+5 \\
00\end{array}$ & $\begin{array}{r}45 \\
83 \\
+5 \\
58\end{array}$ & $\begin{array}{r}51 . \\
38 \\
+5 \\
44\end{array}$ & $\begin{array}{l}55 . \\
55 \\
\pm 3 . \\
51\end{array}$ & $\begin{array}{l}55 . \\
55 \\
\pm 3 . \\
51\end{array}$ & $\begin{array}{c}54 . \\
16 \\
\pm 2 . \\
84\end{array}$ & $\begin{array}{r}41 . \\
66 \\
+3 \\
04\end{array}$ & $\begin{array}{c}38 . \\
88 \\
\pm 3 . \\
51\end{array}$ & $\begin{array}{r}33 . \\
33 \\
\pm 3 . \\
18\end{array}$ & $\begin{array}{r}33 . \\
33 \\
\pm 3 . \\
18\end{array}$ & $\begin{array}{l}31 . \\
94 \\
\pm 1 . \\
38\end{array}$ & $\begin{array}{r}26 . \\
38 \\
\pm 3 \\
34\end{array}$ & $\begin{array}{l}16 . \\
66 \\
\pm 0 . \\
00\end{array}$ & $\begin{array}{c}16 . \\
66 \\
\pm 0 \\
00\end{array}$ & $\begin{array}{r}16 . \\
66 \\
\pm 0 . \\
00\end{array}$ & & $32.06 \pm 2.98$ \\
\hline
\end{tabular}

* Each figure is a mean of 6 observations

* H-High altitude (1700-1800 m asl)

*M-Medium altitude (1600-1700 m asl)

*L-Low altitude (1500-1600 m asl 
Table.3 Per cent incidence of San Jose scale (Quadraspidiotus perniciosus Comstock) on apple at different locations in district Budgam during 2018-2019

\begin{tabular}{|c|c|c|c|c|c|c|c|c|c|c|c|c|c|c|c|c|c|c|c|c|c|c|c|c|c|c|}
\hline \multirow[t]{2}{*}{ Location } & \multirow[t]{2}{*}{ Altitude } & \multicolumn{23}{|c|}{ Standard weeks } & \multirow{2}{*}{$\begin{array}{c}\text { Mean } \\
(\%) \\
\pm \mathrm{SE}\end{array}$} & \multirow{2}{*}{$\begin{array}{c}\text { Cumulative pooled } \\
\text { mean } \\
(\%) \\
\pm \mathrm{SE} \\
\end{array}$} \\
\hline & & $8^{\text {th }}$ & $10^{\text {th }}$ & $12^{\text {th }}$ & $14^{\text {th }}$ & $16^{\text {th }}$ & $18^{\text {th }}$ & $20^{\text {th }}$ & $22^{\text {nd }}$ & $24^{\text {th }}$ & $26^{\text {th }}$ & $28^{\text {th }}$ & $30^{\text {th }}$ & $32^{\text {nd }}$ & $34^{\text {th }}$ & $36^{\text {th }}$ & $38^{\text {th }}$ & $40^{\text {th }}$ & $42^{\text {nd }}$ & $44^{\text {th }}$ & $46^{\text {th }}$ & $48^{\text {th }}$ & $50^{\text {th }}$ & $52^{\text {nd }}$ & & \\
\hline BALPORA & \multirow[t]{2}{*}{ (H) } & $16.66^{*}$ & 16.66 & 16.66 & 16.66 & 16.66 & 16.66 & 16.66 & 24.99 & 24.99 & 33.33 & 33.33 & 33.33 & 33.33 & 33.33 & 33.33 & 33.33 & 16.66 & 16.66 & 16.66 & 16.66 & 16.66 & 16.66 & 16.66 & $22.45 \pm 1.60$ & \multirow[t]{2}{*}{$22.09 \pm 0.36$} \\
\hline KHUDPORA & & 16.66 & 16.66 & 16.66 & 16.66 & 16.66 & 16.66 & 16.66 & 16.66 & 24.99 & 33.33 & 33.33 & 33.33 & 33.33 & 33.33 & 33.33 & 24.99 & 16.66 & 16.66 & 16.66 & 16.66 & 16.66 & 16.66 & 16.66 & $21.73 \pm 1.54$ & \\
\hline NAMBALHAR & \multirow[t]{2}{*}{ (M) } & 16.66 & 16.66 & 16.66 & 16.66 & 16.66 & 16.66 & 24.99 & 33.33 & 33.33 & 33.33 & 33.33 & 33.33 & 33.33 & 33.33 & 33.33 & 33.33 & 24.99 & 16.66 & 16.66 & 16.66 & 16.66 & 16.66 & 16.66 & $23.90 \pm 1.68$ & \multirow[t]{2}{*}{$23.54 \pm 0.36$} \\
\hline KRIMSHORE & & 16.66 & 16.66 & 16.66 & 16.66 & 16.66 & 16.66 & 16.66 & 24.99 & 33.33 & 33.33 & 33.33 & 33.33 & 33.33 & 33.33 & 33.33 & 33.33 & 24.99 & 16.66 & 16.66 & 16.66 & 16.66 & 16.66 & 16.66 & $23.18 \pm 1.65$ & \\
\hline BUGAM & \multirow[t]{2}{*}{ (L) } & 16.66 & 16.66 & 16.66 & 16.66 & 16.66 & 24.99 & 33.33 & 33.33 & 41.66 & 50.00 & 50.00 & 50.00 & 50.00 & 50.00 & 50.00 & 41.66 & 33.33 & 33.33 & 33.33 & 24.99 & 16.66 & 16.66 & 16.66 & $31.88 \pm 2.85$ & \multirow[t]{2}{*}{$31.33 \pm 0.54$} \\
\hline CHADOORA & & 16.66 & 16.66 & 16.66 & 16.66 & 16.66 & 16.66 & 33.33 & 33.33 & 41.66 & 41.66 & 50.00 & 50.00 & 50.00 & 50.00 & 50.00 & 33.33 & 33.33 & 33.33 & 33.33 & 24.99 & 16.66 & 16.66 & 16.66 & $30.79 \pm 2.79$ & \\
\hline $\operatorname{MEAN}(\%) \pm$ SE & & $\begin{array}{l}16.66 \\
\pm 0.00\end{array}$ & $\begin{array}{r}16.66 \\
\pm 0.00\end{array}$ & $\begin{array}{r}16.66 \\
\pm 0.00\end{array}$ & $\begin{array}{l}16.66 \\
\pm 0.00\end{array}$ & $\begin{array}{l}16.66 \\
\pm 0.00\end{array}$ & $\begin{array}{l}18.04 \\
\pm 1.38\end{array}$ & $\begin{array}{l}23.60 \\
\pm 3.34\end{array}$ & $\begin{array}{l}27.77 \\
\pm 2.77\end{array}$ & $\begin{array}{l}33.33 \\
\pm 3.04\end{array}$ & $\begin{array}{r}37.49 \\
\pm 2.84\end{array}$ & $\begin{array}{r}38.88 \\
\pm 3.51\end{array}$ & $\begin{array}{l}38.88 \\
\pm 3.51\end{array}$ & $\begin{array}{r}38.88 \\
\pm 3.51\end{array}$ & $\begin{array}{l}38.88 \\
\pm 3.51\end{array}$ & $\begin{array}{r}38.88 \\
\pm 3.51\end{array}$ & $\begin{array}{l}33.33 \\
\pm 2.15\end{array}$ & $\begin{array}{l}24.99 \\
\pm 3.04\end{array}$ & $\begin{array}{l}22.21 \\
\pm 3.51\end{array}$ & $\begin{array}{l}22.21 \\
\pm 3.51\end{array}$ & $\begin{array}{l}19.43 \\
\pm 1.75\end{array}$ & $\begin{array}{l}16.66 \\
\pm 0.00\end{array}$ & $\begin{array}{l}16.66 \\
\pm 0.00\end{array}$ & \begin{tabular}{|c|}
$16.66 \pm$ \\
0.00
\end{tabular} & & $25.65 \pm 2.86$ \\
\hline
\end{tabular}
* Each figure is a mean of 6 observation

* H-High altitude(1800-1900 m asl)

*L-Low altitude(1600-1700 m asl

Table.4 Per cent incidence of San Jose scale (Quadraspidiotus perniciosus Comstock) at different altitudes on apple in district Baramulla during 2018-2019

\begin{tabular}{|c|c|c|c|c|}
\hline \multirow{2}{*}{ Month } & \multicolumn{3}{|c|}{ Per centincidence } & \multirow{2}{*}{$\begin{array}{c}\text { Mean per cent } \\
\text { incidence }\end{array}$} \\
\cline { 2 - 3 } & High altitude & Medium altitude & Low altitude & $16.66(4.08)^{\mathrm{h}}$ \\
\hline February & $16.66(4.08)^{*}$ & $16.66(4.08)$ & $16.66(4.08)$ & $16.06(4.08)^{\mathrm{h}}$ \\
\hline March & $16.66(4.08)$ & $16.66(4.08)$ & $16.66(4.08)$ & $16.66(4.08)$ \\
\hline April & $16.66(4.08)$ & $16.66(4.08)$ & $16.66(4.08)$ & $16.66(4.08)^{\mathrm{h}}$ \\
\hline May & $20.82(4.56)$ & $24.99(4.99)$ & $35.41(5.94)$ & $27.07(5.17)^{\mathrm{g}}$ \\
\hline June & $33.33(5.77)$ & $35.41(5.95)$ & $50.00(7.07)$ & $40.27(6.26)^{\mathrm{d}}$ \\
\hline July & $40.27(6.34)$ & $47.22(6.87)$ & $64.27(8.07)$ & $50.58(7.09)^{\mathrm{b}}$ \\
\hline August & $\mathbf{5 0 . 0 0 ( 7 . 0 7 )}$ & $\mathbf{5 0 . 0 0 ( 7 . 0 7 )}$ & $\mathbf{6 5 . 5 7 ( 8 . 0 3 )}$ & $\mathbf{5 5 . 1 9 ( 7 . 3 9 ) ^ { \mathrm { a } }}$ \\
\hline
\end{tabular}

$\mathrm{CD}(\mathbf{P}=\mathbf{0 . 0 5})$

Month (A) : (0.121)

Elevation $(B):(0.070)$

Month $\times$ Elevation $(A \times B):(0.191)$

Figure in parentheses are square root transformed values

Values superscripted by same letter (s) are statistically identical 
Table.5 Per cent incidence of San Jose scale (Quadraspidiotus perniciosus Comstock) at different altitudes on apple in district Budgam during 2018-2019

\begin{tabular}{|c|c|c|c|c|}
\hline \multirow{2}{*}{ Month } & \multicolumn{3}{|c|}{ Per centincidence } & Mean per cent \\
& High altitude & Medium altitude & Low altitude & \\
\hline February & $16.66(4.08)^{*}$ & $16.66(4.08)$ & $16.66(4.08)$ & $16.66(4.08)^{\mathrm{f}}$ \\
\hline March & $16.66(4.08)$ & $16.66(4.08)$ & $16.66(4.08)$ & $16.66(4.08)^{\mathrm{f}}$ \\
\hline April & $16.66(4.08)$ & $16.66(4.08)$ & $16.66(4.08)$ & $16.66(4.08)^{\mathrm{f}}$ \\
\hline May & $16.66(4.08)$ & $18.74(4.32)$ & $27.07(5.19)$ & $20.82(4.53)^{\mathrm{e}}$ \\
\hline June & $22.91(4.78)$ & $31.24(5.58)$ & $37.49(6.12)$ & $30.54(5.49)^{\mathrm{c}}$ \\
\hline July & $33.33(5.77)$ & $33.33(5.77)$ & $48.61(6.97)$ & $38.42(6.17)^{\mathrm{a}}$ \\
\hline August & $\mathbf{3 3 . 3 3 ( 5 . 7 7 )}$ & $\mathbf{3 3 . 3 3 ( 5 . 7 7 )}$ & $\mathbf{5 0 . 0 0 ( 7 . 0 7 )}$ & $\mathbf{3 8 . 8 8 ( 6 . 2 0})^{\mathrm{a}}$ \\
\hline September & $31.24(5.58)$ & $33.33(5.77)$ & $43.74(6.61)$ & $36.10(5.99)^{\mathrm{b}}$ \\
\hline October & $16.66(4.08)$ & $20.82(4.56)$ & $33.33(5.77)$ & $23.60(4.80)^{\mathrm{d}}$ \\
\hline November & $16.66(4.08)$ & $16.66(4.08)$ & $29.16(5.40)$ & $20.82(4.52)^{\mathrm{e}}$ \\
\hline December & $16.66(4.08)$ & $16.66(4.08)$ & $16.66(4.08)$ & $16.66(4.08)^{\mathrm{f}}$ \\
\hline Mean & $22.07(4.69)^{\mathrm{C}}$ & $23.74(4.87)^{\mathrm{B}}$ & $31.93(5.65)^{\mathrm{A}}$ & \\
\hline
\end{tabular}

\section{$\mathrm{CD}(\mathrm{P}=\mathbf{0 . 0 5})$}

Month (A) : (0.142)

Elevation (B) : (0.082)

Month $\times$ Elevation $(\mathrm{A} \times \mathrm{B}):(\mathbf{0 . 2 2 4})$

Figure in parentheses are square root transformed values

Values superscripted by same letter (s) are statistically identical

Table.6 Correlation between incidence of San Jose scale (Quadraspidiotus pernisiocus) and weather parameters during 2018-2019

\begin{tabular}{|c|c|c|}
\hline \multirow{2}{*}{ Weather parameters } & Baramulla & Budgam \\
\cline { 2 - 3 } & Incidence & Incidence \\
\hline Maximum temperature & $0.757 * *$ & $0.732^{* *}$ \\
\hline Minimum temperature & -0.202 & -0.072 \\
\hline Rainfall & 0.156 & 0.112 \\
\hline Relative humidity morning & $0.752^{* *}$ & $0.730^{* *}$ \\
\hline Relative humidity evening & -0.250 & -0.292 \\
\hline Sunshine hour & $0.571^{*}$ & $0.506^{*}$ \\
\hline
\end{tabular}


Table.7 Regression coefficient $\left(\mathrm{R}^{2}\right)$ between incidence of San Jose scale (Quadraspidiotus pernisiocus) and weather parameters along with coefficient of Determination in district

Baramulla during 2018-2019

\begin{tabular}{|c|c|c|c|}
\hline Regression equation & $\mathbf{R}^{2}$ & $100 \mathbf{R}^{2}$ & Difference \\
\hline$Y=6.078+1.260 X_{1}$ & 0.5659 & 56.59 & 56.59 \\
\hline$Y=33.218-0.941 X_{1}+2.702 X_{2}$ & 0.5668 & 56.68 & 0.09 \\
\hline$Y=53.186-2.128 X_{1}+4.050 X_{2}-1.559 X_{3}$ & 0.5830 & 58.30 & 1.62 \\
\hline$Y=-75.978+0.354 X_{1}+1.957 X_{2}+0.127 X_{3}+1.056 X_{4}$ & 0.8131 & 81.31 & 23.01 \\
\hline $\begin{array}{c}Y=-174.730+2.205 X_{1}+0.823 X_{2^{-}} \\
1.851 X_{3}+0.800 X_{4}+1.628 X_{5}\end{array}$ & 0.8236 & 82.36 & 1.05 \\
\hline $\begin{array}{c}Y=-166.330+2.718 X_{1}+0.608 X_{2^{-}} \\
1.914 X_{3}+0.693 X_{4}+1.609 X_{5}-1.460 X_{6}\end{array}$ & 0.9909 & 99.09 & 16.73 \\
\hline
\end{tabular}

$\mathrm{X} 1=$ maximum temperature, $\mathrm{X} 2=$ minimum temperature, $\mathrm{X} 3=$ rainfall, $\mathrm{X} 4=$ relative humidity morning, $\mathrm{X} 5=$ relative humidity evening, $\mathrm{X} 6=$ sunshine hour.

Table.8 Regression coefficient $\left(\mathrm{R}^{2}\right)$ between incidence of San Jose scale (Quadraspidiotus pernisiocus) and weather parameter along with coefficient of Determination in district Budgam during 2018-2019

\begin{tabular}{|c|c|c|c|}
\hline Regression equation & $\mathbf{R}^{2}$ & $100 \mathbf{R}^{2}$ & Difference \\
\hline$Y=6.719+0.899 X_{1}$ & 0.6376 & 63.76 & 63.76 \\
\hline$Y=21.662-0.312 X_{1}+1.487 X_{2}$ & 0.6471 & 64.71 & 0.95 \\
\hline$Y=48.377-1.901 X_{1}+3.291 X_{2}-2.086 X_{3}$ & 0.6643 & 66.43 & 1.72 \\
\hline$Y=-24.378-0.502 X_{1}+2.113 X_{2}-1.135 X_{3}+0.595 X_{4}$ & 0.8407 & 84.07 & 17.64 \\
\hline $\begin{array}{c}Y=-63.942+0.238 X_{1}+1.658 X_{2-} 1.928 X_{3}+0.492 X_{4}+ \\
0.652 X_{5}\end{array}$ & 0.8472 & 84.72 & 0.65 \\
\hline $\begin{array}{c}Y=-28.613+2.397 X_{1}+0.752 X_{2^{-}} \\
2.192 X_{3}+0.0419 X_{4}+0.572 X_{5}-6.142 X_{6}\end{array}$ & 0.9791 & 97.91 & 13.19 \\
\hline
\end{tabular}

$\mathrm{X} 1=$ maximum temperature, $\mathrm{X} 2=$ minimum temperature, $\mathrm{X} 3=$ rainfall, $\mathrm{X} 4=$ relative humidity morning, $\mathrm{X} 5=$ relative humidity evening, $\mathrm{X} 6=$ sunshine hour.

\section{References}

Anonymous, 2018. District wise area and production of major horticultural crops in Jammu and Kashmir state for the year 2018. Department of Horticulture, Jammu and Kashmir.

Anonymous.2019. Area and production of major horticultural crops for $\mathrm{J}$ and $\mathrm{K}$. Directorate of Horticulture Kashmir.

Balusu, R. R., Palmer, K. R., Cofer, T., Pitts, J. A., Horton, D. L. and Fadamiro, H.
Y. 2017.Evaluation of insecticides for control of San Jose scale in Alabama Peaches. Arthropod Management Tests 42(1): 1-2.

Bandey,S.A., Sharma, R. and Singh, A. 2012. Diversity of apple pests and their effects on the productivity of apple crops in Jammu region of $\mathrm{J} \& \mathrm{~K}$ state. International Journal of Advanced Biotechnology Research, 2(2): 367-369.

Botha, A. 2008.The population dynamics and its role in controlling Quadraspidiotus 
perniciosus by Aphytisproclia. Supriadi, K., Mudijiono, G., Abadi, A.L. and Bulgarian Journal of Agricultural Karindah, S. 2015. The Influence of Science 1(2): 250-252.

Rather, A. Q. and Masoodi, M. A. 1994. Studies on the ecobiology, distribution and host range of Panonychusulmi in Kashmir. Journal of Ecobiology, 6(4): 287-91. Environmental Factors to The Abundance of Scales (Hemiptera: Diaspididae) Population on Apple Crop. The Journal of Tropical Life Science, 5(1): 20-24.

\section{How to cite this article:}

Insha Hamid, A.R. Wani, M.A. Sofi, S.S. Pathania, Nageena nazir, Mohd. Saleem Mir and Hassan, G.I. 2021. Incidence of San Jose Scale, (Quadraspidiotus perniciosus Comstock) in Relation to Abiotic Factors on Apple Tree. Int.J.Curr.Microbiol.App.Sci. 10(01): 1730-1738. doi: https://doi.org/10.20546/ijcmas.2021.1001.203 